\title{
BMJ Open PrEP Implementation for Mothers in Antenatal Care (PrIMA): study protocol of a cluster randomised trial
}

\author{
Julia C Dettinger, ${ }^{1}$ John Kinuthia, ${ }^{2}$ Jillian Pintye, ${ }^{1}$ Nancy Mwongeli, ${ }^{2}$ \\ Laurén Gómez, ${ }^{1}$ Barbra A Richardson, ${ }^{1,3}$ Ruanne Barnabas, ${ }^{1,4}$ Anjuli D Wagner, ${ }^{\oplus 1}$ \\ Gabrielle O'Malley, ${ }^{1}$ Jared M Baeten, ${ }^{1,4,5}$ Grace John-Stewart ${ }^{1,4,5,6}$
}

To cite: Dettinger JC, Kinuthia J, Pintye J, et al. PrEP Implementation for Mothers in Antenatal Care (PrIMA): study protocol of a cluster randomised trial. BMJ Open 2019;9:e025122. doi:10.1136/ bmjopen-2018-025122

- Prepublication history for this paper is available online. To view these files, please visit the journal online (http://dx.doi. org/10.1136/bmjopen-2018025122).

Received 30 June 2018 Revised 8 January 2019 Accepted 11 January 2019

Check for updates

(c) Author(s) (or their employer(s)) 2019. Re-use permitted under CC BY-NC. No commercial re-use. See rights and permissions. Published by BMJ.

${ }^{1}$ Department of Global Health, University of Washington, Seattle, Washington, USA ${ }^{2}$ Department of Research and Programs, Kenyatta National Hospital, Nairobi, Kenya

${ }^{3}$ Department of Biostatistics, University of Washington, Seattle, Washington, USA ${ }^{4}$ Department of Medicine, University of Washington, Seattle, Washington, USA ${ }^{5}$ Department of Epidemiology, University of Washington, Seattle, Washington, USA

${ }^{6}$ Department of Pediatrics, University of Washington, Seattle, Washington, USA

Correspondence to Ms Julia C Dettinger; jcdettin@uw.edu

\section{ABSTRACT}

Introduction Women in regions with high HIV prevalence are at high risk of HIV acquisition during pregnancy and postpartum, and acute maternal HIV contributes a substantial proportion of infant HIV infections. Pre-exposure prophylaxis (PrEP) could prevent HIV during pregnancy/postpartum; however, identifying women who would most benefit from PrEP in this period is challenging. Women may not perceive risk, may not know partner HIV status and partners may have external partners during this period. PrEP offer in pregnancy could be universal or risk guided.

Methods and analysis The PrEP Implementation for Mothers in Antenatal Care (PrIMA) study is a cluster randomised trial that aims to determine the best model for PrEP implementation in pregnancy, among women attending public sector maternal child health clinics in Western Kenya (HIV prevalence $>25 \%$ ). Twenty clinics are randomised to either universal PrEP offer following standardised counselling ('Universal arm' 10 clinics) or risk screening with partner self-test option ('Targeted arm' 10 clinics). Four thousand women will be enrolled and followed through 9-month postpartum. The primary analysis will be intention to treat. Outcomes reflect the balance between HIV preventive effectiveness and avoiding unnecessary PrEP exposure to women at low risk and include: maternal HIV incidence, PrEP uptake, PrEP adherence, PrEP duration, 'appropriate' PrEP use (among women with objective evidence of potential risk), infant birth outcomes, infant growth and partner selftesting uptake. To better understand the feasibility and acceptability of the provision of PrEP in these settings, qualitative interviews and cost-effectiveness analyses will be conducted.

Ethics and dissemination The protocol was approved by the institutional review boards at Kenyatta National Hospital and the University of Washington. An external advisory panel monitors adverse and social harm events. Results will be disseminated through peer-reviewed journals, presentations at local and international conferences to national and global policy makers, community and participants.

Trial registration number NCT03070600.

\section{INTRODUCTION}

In high HIV prevalence settings, women have high risk of HIV acquisition during

\section{Strengths and limitations of this study}

- The cluster randomised trial design enables rigorous concurrent comparison of two feasible pre-exposure prophylaxis (PrEP) implementation models in maternal child health clinics that could generalise to other high HIV prevalence settings.

- This study will explore PrEP uptake, adherence, HIV incidence and maternal/infant outcomes among pregnant women offered PrEP who have been understudied in previous PrEP research.

- Comprehensive data collection including psychosocial factors, self-efficacy, partnership characteristics and HIV risk characteristics will increase understanding of the factors that influence PrEP uptake and adherence.

- Collection of dried blood spots will allow for objective assessment of PrEP adherence through measurement of tenofovir disoproxil fumarate concentrations in blood.

- If PrEP uptake and use are appropriate in both arms, HIV incidence may be equivalently low, promoting deeper exploration of cost and acceptability in order to make recommendations regarding which model is best suited for adoption within the health system.

pregnancy and postpartum. A meta-analysis of 19 studies found high HIV incidence in pregnant and postpartum. ${ }^{1}$ A 2015 study in Western Kenya found an HIV incidence rate of 2.31 infections/1000 person years among pregnant and postpartum women. ${ }^{2}$ A recent analysis among women in HIV serodiscordant partnerships, in seven African countries including Kenya, demonstrated that the risk of HIV acquisition per coital act was higher in late pregnancy and postpartum compared with non-pregnant women. ${ }^{3}$ Among mothers acutely infected with HIV, there is a twofold increase in the risk of mother-to-child HIV transmission (MTCT) compared with chronic HIV maternal infection due to higher viral load and absent HIV immune responses. ${ }^{4}$ Prevention of MTCT (PMTCT) programmes 
effectively identify women with chronic HIV infections and implement antiretroviral treatment (ART). However, current PMTCT programmes may miss acute HIV infections occurring in pregnancy or postpartum. As a result, acute HIV infections during pregnancy contribute an increasing proportion of infant HIV infections. Over $40 \%$ of new infant HIV infections worldwide are estimated to be due to maternal HIV acquisition in pregnancy and postpartum. ${ }^{5}$ Given the high HIV incidence in pregnancy/postpartum and the risk of transmission to the infant, there is need for effective HIV prevention in this critical period.

Oral pre-exposure prophylaxis (PrEP) with tenofovir disoproxil fumarate (TDF) and emtricitabine-triphosphate (FTC) is a highly effective intervention for HIV prevention. ${ }^{6}$ TDF and FTC have been evaluated for safety among both women receiving PrEP and HIV-positive women receiving ART for PMTCT. ${ }^{78}$ A systematic review concluded that there is no safety-related rationale for prohibiting PrEP during pregnancy and/or breast feeding. ${ }^{7}$ The review found no statistically significant differences in stillbirth/pregnancy loss, preterm delivery, low birth weight, small for gestational age, birth defects and infant or maternal mortality among women using TDF versus those not on TDF. ${ }^{7}$ Additionally, there is minimal penetration of tenofovir (the metabolite of TDF) into breastmilk. ${ }^{9}$ Finally, PrEP programmes into pregnant and postpartum populations include frequent HIV testing, facilitating early identification of incident HIV infections and subsequent ART initiation. ${ }^{10}$ Based on these data, the WHO recommends TDF/FTC-based oral PrEP for pregnant and breastfeeding women at high risk for HIV as a female-controlled prevention strategy. ${ }^{11}$

While safety data are generally reassuring, determining which pregnant women are most likely to benefit from PrEP and prioritising them for PrEP delivery would have multiple benefits, including reducing unnecessary PrEP exposure among women who are not at risk for acquiring HIV and maximising cost-effectiveness. A universal offer of PrEP would allow women to choose whether they wanted to use PrEP. Universal access to PrEP has shown high uptake among men who have sex with men in high-income settings, with evidence that those who elect to start PrEP are at particularly high risk. ${ }^{12}{ }^{13}$ However, some have suggested that risk-scoring tools could increase efficiency by helping clinicians identify those at high risk, limiting PrEP offer to the subset with objective markers of risk. ${ }^{14}$ In addition, for individuals who may be unaware of their risk, which is often the case for pregnant women, risk evaluation approaches may reveal previously unperceived risk. For pregnant women, the WHO recommends using risk-guided approaches for HIV prevention as part of a comprehensive PMTCT package. ${ }^{11}$ A risk scoring tool to identify high HIV risk pregnant women and using a simple set of easily accessible variables (partner HIV status unknown, number of lifetime sexual partners and history of syphilis) was developed and validated using data from 1304 Kenyan women. ${ }^{15}$ Women with a score $>6$ made up $16 \%$ of the population but accounted for $56 \%$ of HIV acquisition events. This or a similar tool may be specifically useful for optimising PrEP delivery for pregnant and postpartum women.

In Western Kenya, with $>15 \%$ HIV prevalence, there is an urgent need to identify effective strategies for preventing acute HIV infections in pregnancy and postpartum. ${ }^{16}$ This cluster randomised trial will compare two models for PrEP delivery within antenatal care (ANC) clinics in Kenya. One model is universal offer of PrEP to all pregnant women, and the other is targeted offer (using risk tool and offering partner HIV self-test services to refine risk guidance). We hypothesise that because of better matching of PrEP use to HIV risk, targeted PrEP approaches will result in lower HIV incidence and optimised proportion of women on PrEP. We predict that this targeted PrEP approach will result in more 'appropriate' PrEP use, better adherence, shorter PrEP duration (stop after partner viral suppression), more partners on ART and similar infant outcomes.

\section{METHODS/DESIGN \\ Study design}

The PrEP Implementation for Mothers in Antenatal Care (PrIMA) study aims to determine the best model for PrEP delivery in pregnancy in public sector maternal child health $(\mathrm{MCH})$ clinics. ${ }^{17}$ The study is a cluster randomised comparison of two models of PrEP delivery in ANC.

\section{Interventions}

Two PrEP implementation models will be compared. In clinics randomised to universal PrEP counselling arm (universal arm), all enrolled women receive standardised PrEP counselling and self-select whether they want to initiate PrEP. In clinics randomised to targeted PrEP counselling (targeted arm), study participants undergo a risk assessment, and those who are identified as being at high risk for HIV acquisition based on the Pintye et $a l^{15}$ risk score receive PrEP counselling and then decide whether they want to initiate PrEP. High risk is defined as either a risk score greater than 6 or any of the risk factors outlined by the Kenya National AIDS and STI Control Programme (NASCOP), including: transactional sex, recent STI, sex against their will, intimate partner violence, intravenous drug use and recurrent use of postexposure prophylaxis. ${ }^{10} 15$ In addition, targeted arm participants receive HIV self-test kits to take to partners for partner HIV testing to further enhance estimation of potential HIV risk.

\section{Study sites and population}

Facility selection process

Facilities were selected based on discussions with Kenya NASCOP and the local county governments of Homa Bay and Siaya. Facilities were considered for inclusion if they were located in a county with greater than $20 \%$ HIV seroprevalence, have $>350 \mathrm{HIV}$-negative clients attending 


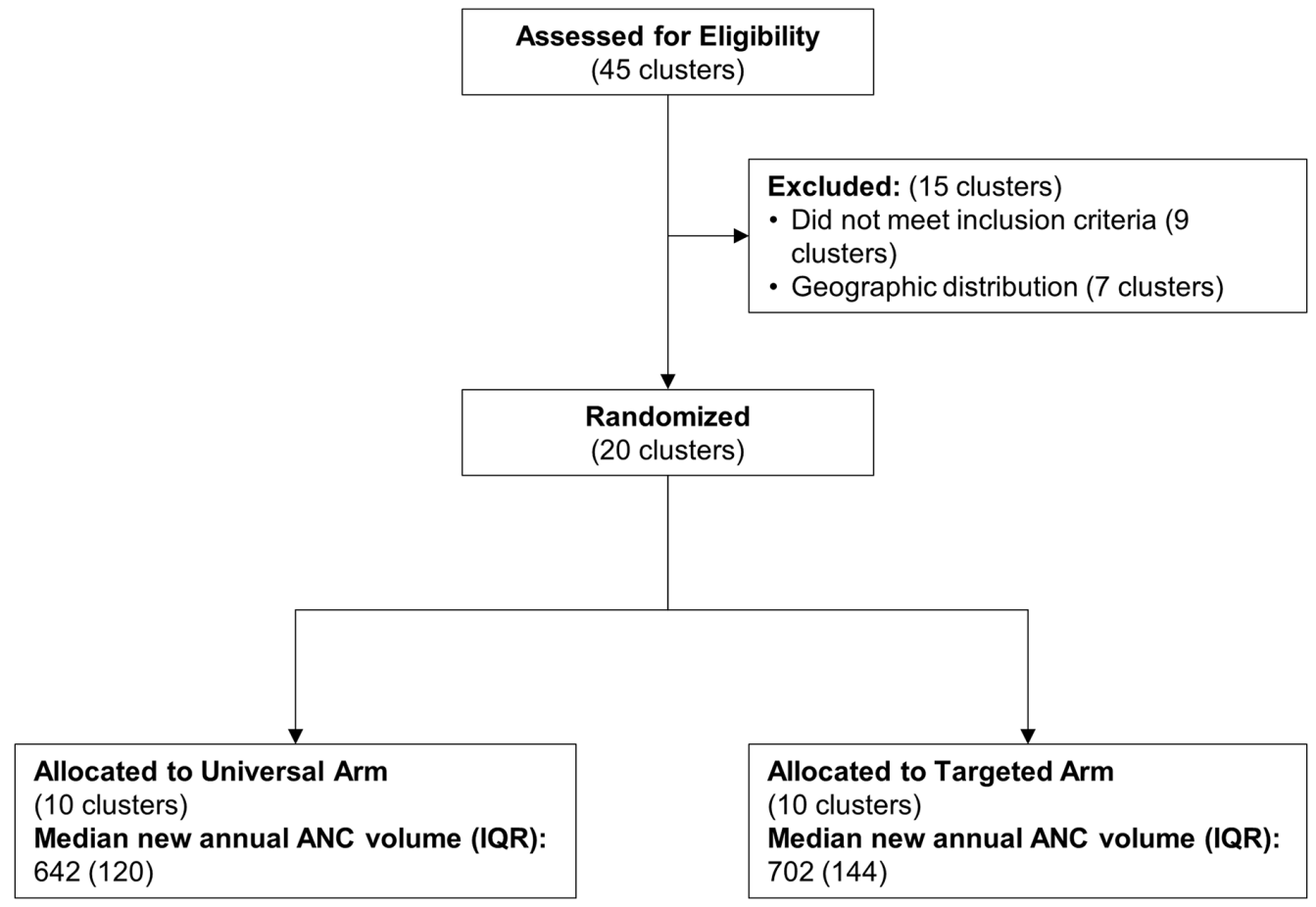

Figure 1 Adapted CONSORT diagram for cluster randomised trials. ANC, antenatal care; CONSORT, Consolidated Standards of Reporting Trials.

ANC per year and offer postnatal care services including infant immunisations. Forty-five facilities were evaluated for inclusion in the PrIMA study, of which 30 met the inclusion criteria. Among the 30 eligible facilities, 20 were selected, with input from the County Ministry of Health, to ensure geographic distribution such that at least one facility per subcounty was included in the study (figure 1). This geographic distribution also ensured that facilities were dispersed over wide distances to avoid contamination between study sites. The 20 sites are dispersed over $300 \mathrm{~km}$ (figure 2).

\section{Individual eligibility criteria}

Facilities enrol at least 200 HIV-negative women attending ANC at the facility. Eligibility for enrolment include: women who are pregnant, HIV negative, not currently using PrEP, age $\geq 15$ years (the age of emancipation in pregnancy in Kenya), tuberculosis negative, plan to reside

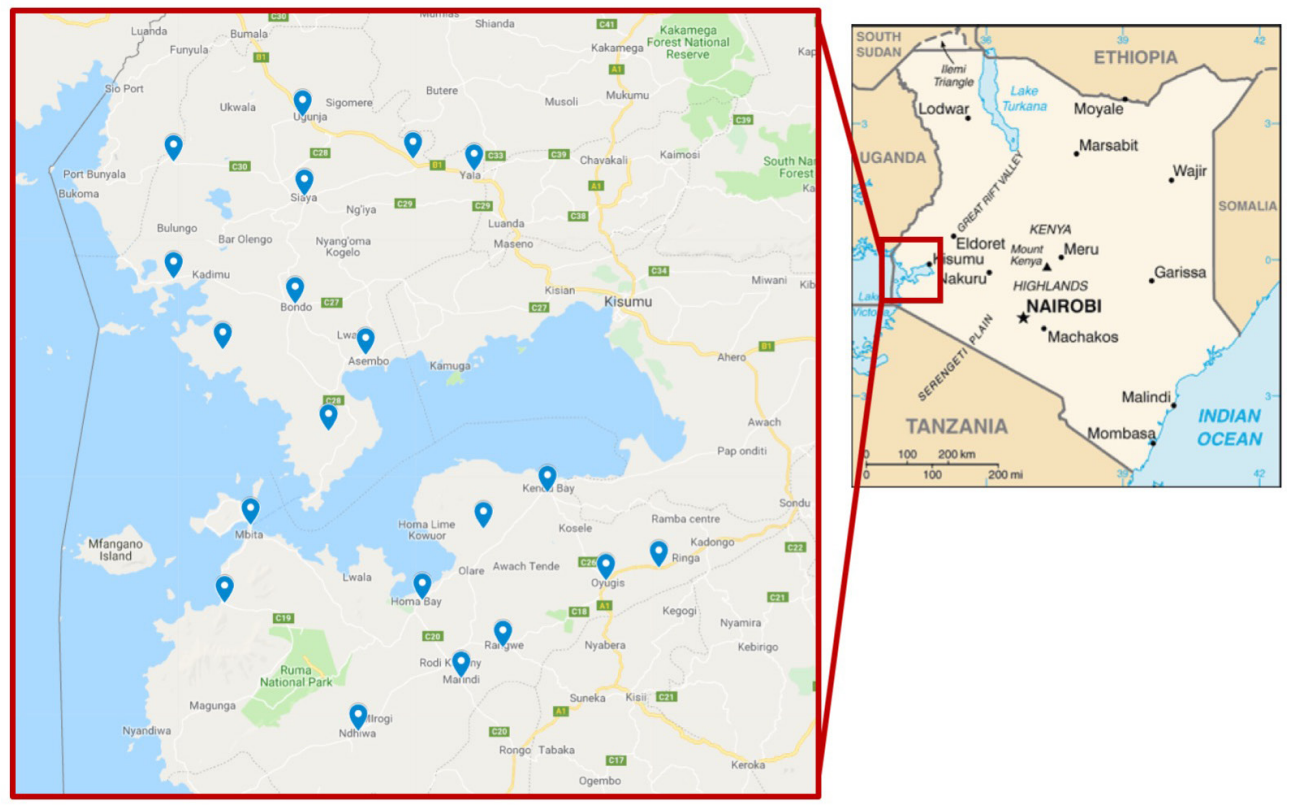

Figure 2 PrIMA study sites. PrIMA, PrEP Implementation for Mothers in Antenatal Care. 
Table 1 Summary group characteristics for PrIMA restricted randomisation

\begin{tabular}{llllll}
\hline Group & County & $\begin{array}{l}\text { County HIV prevalence, } \\
\mathbf{2 0 1 6}^{\mathbf{1 6}} \mathbf{( \% )}\end{array}$ & $\begin{array}{l}\text { No. of } \\
\text { facilities }\end{array}$ & $\begin{array}{l}\text { Mean ANC } \\
\text { Volume (SD) }\end{array}$ & Group description \\
\hline 1 & Homa Bay & 26.0 & 2 & $173(60.8)$ & High volume \\
2 & Homa Bay & 26.0 & 4 & $59.8(1.3)$ & Medium volume \\
3 & Homa Bay & 26.0 & 4 & $53.8(3.3)$ & Low volume \\
4 & Siaya & 24.8 & 2 & $140(26.9)$ & High volume \\
5 & Siaya & 24.8 & 4 & $54.8(6.4)$ & Medium volume \\
\hline 6 & Siaya & 24.8 & 4 & $38(5.5)$ & Low volume \\
\hline
\end{tabular}

ANC, antenatal care; PrEP, pre-exposure prophylaxis; PrIMA, PrEP Implementation for Mothers in Antenatal Care.

in area for at least 1-year postpartum, plan to receive postnatal and infant care at the study facility and who are not currently enrolled in any other studies.

\section{Randomisation}

The facility-level cluster randomisation was conducted using a restricted randomisation approach based on ANC volume and stratified by county. ${ }^{18}$ Prior to randomisation, each facility was categorised by county and ANC volume and assigned to one of six groups (table 1). To build camaraderie and demonstrate transparency of the randomisation process, PrIMA staff at each facility were asked to select a ball from a bag within each of the six groups to ensure balance in county and ANC volume. The bag contained balls of two different colours, with 20 balls overall (10 of each colour). Through this procedure, each facility was randomly assigned to a study arm.

\section{Blinding}

Since the randomisation for PrIMA occurs at the facility level, it is impossible to blind study team members or participants to the randomisation assignments. However, procedures to minimise the influence of the unblinded nature of this study on outcomes have been implemented. Ongoing data monitoring does not include information about study endpoints disaggregated by facility or study arm. Only the study biostatistician reviews data on study on PrEP uptake or adherence by study arm or facility. These reports are reviewed, as necessary, during closed sessions of the External Advisory Panel (EAP), excluding study team members who are implementing the study.

\section{Study procedures}

Aim 1: a cluster randomised clinical trial (RCT) to assess PrEP uptake and outcomes between two PrEP delivery strategies

Study timeline

Enrolment for the PrIMA study was initiated on 15 January 2018 with a targeted enrolment completion of 30 April 2019. It is anticipated that the final study participants will exit the study by the end of 2020 .

\section{Participant screening and enrolment}

Women are approached while waiting for ANC visits at each facility and invited to undergo screening for eligibility into the PrIMA Study. Information on facility volume, clients approached, clients screened and participants enrolled is reported daily by study nurses. All eligible clients undergo a written informed consent process by PrIMA staff in the facility prior to initiation of study procedures.

\section{Enrolment visit data collection}

At enrolment, questionnaires are administered to ascertain demographics, mental health, social support systems, drug and alcohol use, risk perception, self-efficacy, risk assessment, partner HIV status and prior obstetric history. Data from the $\mathrm{MCH}$ card are abstracted, including confirmation of HIV and syphilis test results. In both arms, the risk assessment information is used to characterise the populations during the analysis phase; however, in the targeted arm, this information is also used in real-time to guide PrEP provision.

\section{Universal PrEP clinics and enrolment visit}

Counselling at universal arm facilities uses a standardised individual counselling script that includes information that PrEP is available for women at risk for HIV, PrEP can be perscribed at this clinic, that HIV prevalence in the region is high, and to highlight that women with HIV positive partners or who do not know their partner's status may be at risk for acquiring HIV. Counselling specifies that women may have their own reasons to feel at risk or to want PrEP. Following this standardised counselling, women decide whether to accept a PrEP assessment using the Ministry of Health (MOH) PrEP Card. Participants may elect to deliberate on the decision and return for a PrEP evaluation at a subsequent study visit. Participants who meet the clinical eligibility requirements for PrEP under Kenya PrEP guidelines (weight: $>35 \mathrm{~kg}$, age: $>15$ years, creatinine clearance $[\mathrm{CrCl}]: \geq 50 \mathrm{~mL} / \mathrm{min}$ ) are initiated on PrEP. NASCOP guidelines for PrEP initiation and follow-up are described in detail below. Following the clinical components of the enrolment visit, all participants complete the enrolment CRFs (figure 3).

\section{Targeted PrEP clinics and enrolment visit}

The targeted arm clinics provide two inter-related innovations: risk assessment-informed PrEP counselling and partner HIV self-test counselling. ${ }^{19}$ 

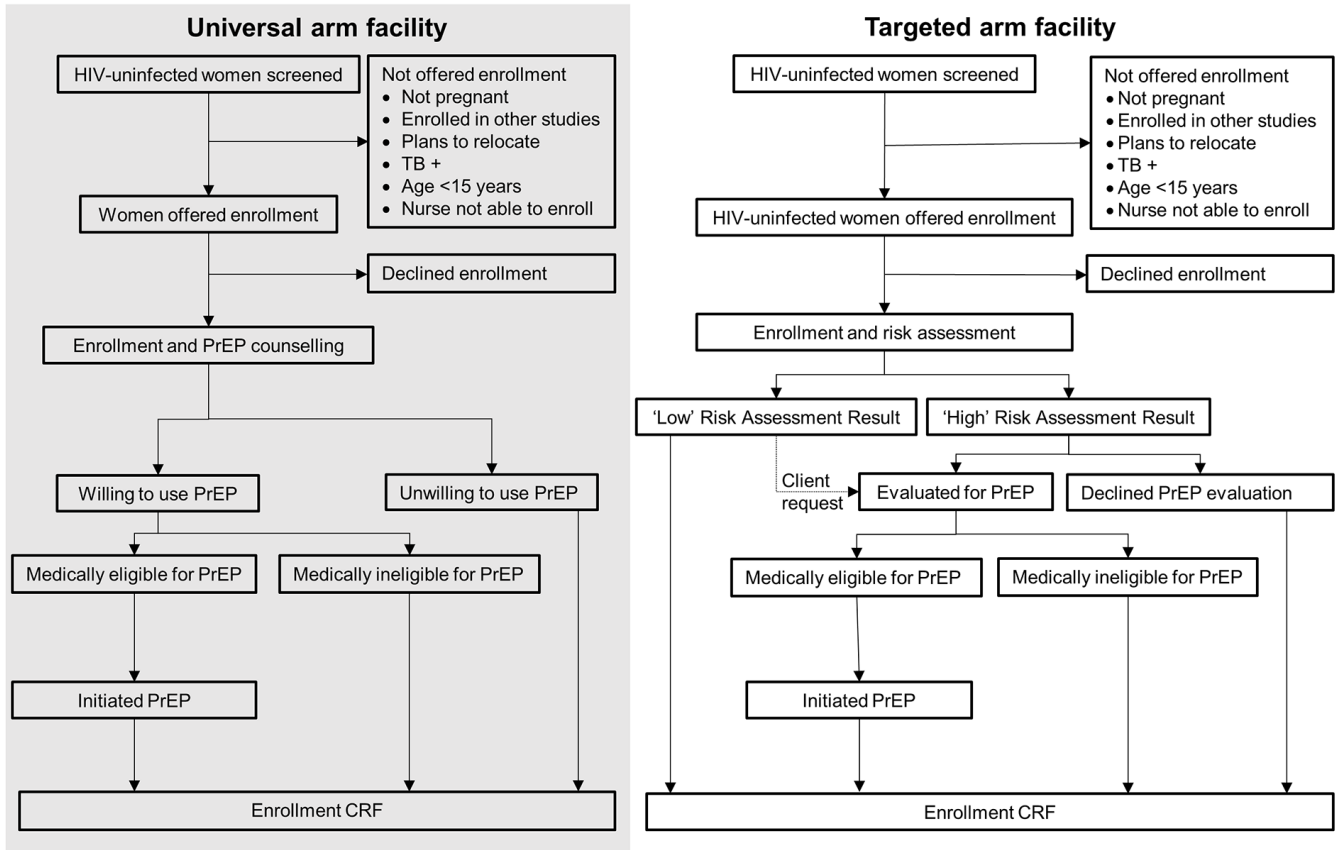

Figure 3 PrIMA enrolment visit procedures by study arm, and interventions and assessments throughout the study. PrEP, preexposure prophylaxis; PrIMA, PrEP Implementation for Mothers in Antenatal Care.

Risk assessment and PrEP counselling: PrEP risk assessment guides PrEP counselling. Participants who are identified as being at risk using a combination of $\mathrm{MOH}$ rapid assessment tools and a validated risk assessment for pregnant women receive additional PrEP counselling that includes information about the specific risk factors identified through the risk assessment (figure 4). These participants then elect whether to proceed to the clinical eligibility assessment. Participants

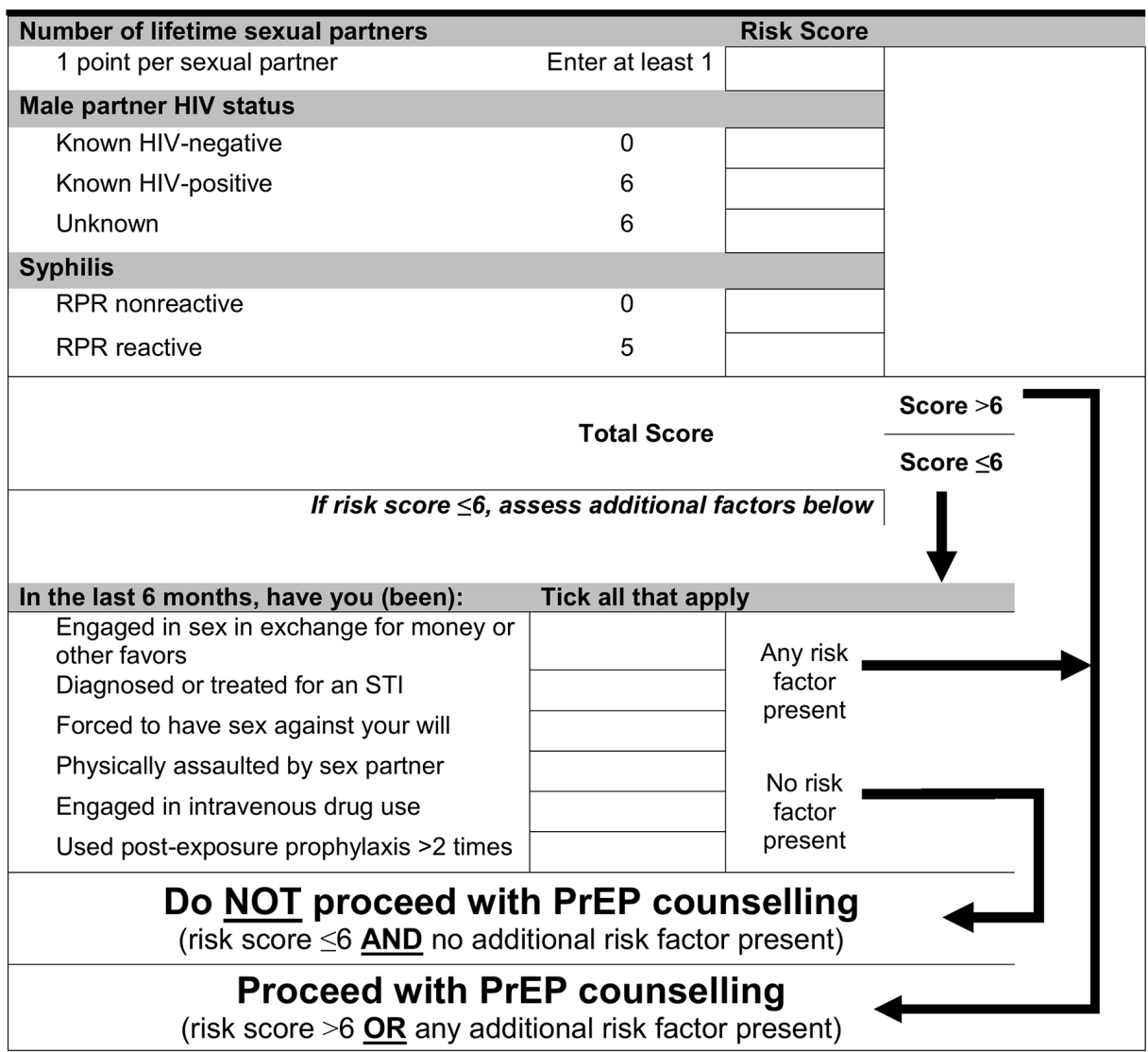

Figure 4 Risk assessment for targeted arm participants. PrEP, pre-exposure prophylaxis. 
who do not meet the risk assessment criteria are not counselled on PrEP. However, if they ask for PrEP, they enter the clinical assessment pathway and can be prescribed PrEP if found to be clinically eligible (figure 3 ). Women who request PrEP despite not meeting the targeted PrEP criteria will be identified and approached for in-depth interviews to better understand their choices.

Partner HIV self-testing: women in targeted arm facilities are also offered HIV self-test kits to take to their partner for HIV testing. This is used to provide further information on partner HIV status and further define the participant's risk. At follow-up visits, participants report the results of partner self-test results. These results inform the decision to either initiate women in previously unidentified serodiscordant couples on PrEP or to recommend women discontinue PrEP if their partner is HIV negative.

Trained programme staff counsel study participants in the use of self-testing kits including that the use of the test kits is voluntary and they can change their mind at any time. PrIMA nurses first assess whether the women believe violence could occur when providing the self-testing kit to their partner. Women who are comfortable providing a self-test to their sexual partner(s) are instructed by study staff on the use of an oral fluid-based rapid HIV test (OraQuick Rapid HIV-1/2 antibody test, OraSure Technologies, Bethlehem, Pennsylvania, USA). All women are offered at least two self-tests so that they can take the test at the same time as their partner. Study staff demonstrate how to use the oral self-test. Women report on the results of their partner's self-test at their next study visit. These activities follow the same procedures as other partner HIV self-test kit distribution studies in the region, which have been shown to have low rates of adverse events. ${ }^{19}$

\section{PrEP initiation and follow-up, universal and targeted arms}

PrEP medication and dosing follows the 2016 Guidelines on Use of Antiretroviral Drugs for Treating and Preventing HIV Infections in Kenya using the preferred oral TDF/FTC $(300 \mathrm{mg} / 200 \mathrm{mg})$ once per day. ${ }^{10}$ Among women who start PrEP, instructions on use of drug and counselling to optimise adherence are identical between targeted and universal clinics. Any drug-related reactions, adverse events or social harm events are documented.

Women who elect to start PrEP and are clinically eligible at any point in the study receive point of care hepatitis B (HBsAg) using HBsAg One Step Hepatitis Surface Antigen Test Strip and creatinine (Cr) testing using Nova StatSensor Xpress Creatinine point-of-care handheld analyser. ${ }^{20}$ Women who are HBsAg positive are referred for comanagement of hepatitis B and allowed to initiate PrEP use. Women who have estimated $\mathrm{CrCl}$ $\leq 50 \mathrm{~mL} / \mathrm{min}$ are identified as clinically ineligible to initiate PrEP. Maternal Cr among PrEP users is obtained annually, and $\mathrm{CrCl}$ is estimated. PrEP is discontinued if (1): participant seroconverts during the study; (2) participant's risk status changes (eg, a partner who previously refused or was unable to take the self-test tests negative); (3) renal dysfunction with $\mathrm{CrCl}<50 \mathrm{~mL} / \mathrm{min}$; (4) participant requests to stop; (5) sustained non-adherence; or (6) participant reports their HIV-positive partner has achieved sustained viral suppression. Women who seroconvert are managed according to NASCOP guidelines including collection of dried blood spots (DBS) for resistance testing and referral for follow-up care including prompt provision of ART for PMTCT.

\section{Data collection at follow-up visits, universal and targeted arms}

All study visits align with the Kenya MCH schedule for routine ANC and postpartum/immunisation visits: monthly for ANC until delivery and then 6-week, 14-week, 6-month and 9-month postpartum (table 2). Participants receive HIV rapid testing at all study visits and Ksh 300 to compensate for their time and transportation expenses to participate in the study. Retention officers provide reminders about study visits to participant. If needed, retention officers may visit participants in their homes to encourage retention.

Given the possibility of differential lost to follow-up between study arms, retention officers will engage with all study participants at all study visits in both RCT arms. They will encourage all participants to come in for study visits, and study visit windows are structured so that a participant could return at any point between enrolment and 9-month postpartum and fall within a study visit window.

Data on risk assessment, partnership characteristics and sociobehavioural factors are collected at selected study follow-up visits. Additional data are collected from participant self-report or abstracted from MCH cards on infant growth, birth length, birth outcomes (stillbirth, miscarriage, mortality and congenital anomalies), birth weight, estimated gestation at birth or diagnosed preterm birth and serial growth. Z scores for infant growth will be calculated using WHO Anthro-Plus. ${ }^{21}$

A combination of self-report and tenofovir-diphosphate (TFV-DP) and emtricitabine-triphosphate (FTC-TP) in red blood cells from DBS are used to assess PrEP adherence. DBS is collected, using a finger stick protocol, from all women on PrEP at all follow-up visits. A subset of DBS specimens from all women on PrEP will be randomly selected for testing. DBS testing will be conducted using validated liquid chromatography/tandem mass spectrometry assays. ${ }^{22}{ }^{23}$ The presence of detectable FTC-TP is a proxy for recent adherence in the preceding 48 hours. Given a half-life of 17 days for TFV-DP in DBS, levels of TFV-DP per punch can approximate cumulative dosing (<349 fmol/punch: <2 tabs/week; 350-699 fmol/punch: 2-3 tabs/week; 700-1249 fmol/punch: 4-6 tabs/week; and $1250 \mathrm{fmol} /$ punch (daily dosing) ${ }^{22}{ }^{23}$ The levels used in this analysis will be updated if evidence emerges that other levels are more predictive.

\section{Data management}

Clinical and baseline data collected during the course of this study are collected electronically via RedCap data collection software.$^{24}$ Data are uploaded daily via RedCap Mobile Application from Android tablets to the RedCap 
Table 2 Study timeline including randomisation, enrolment and follow-up visits

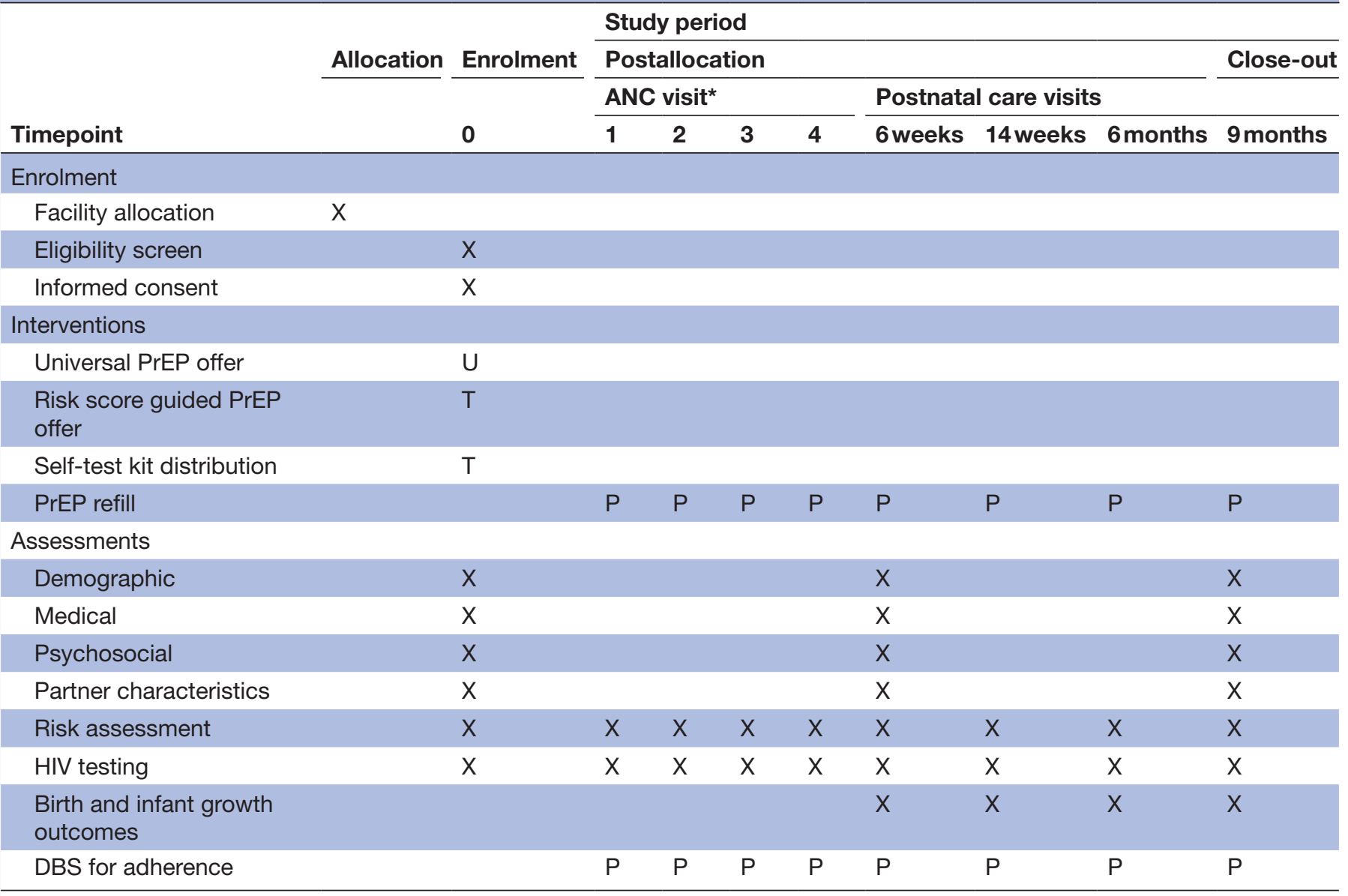

*Number of ANC visits depends on gestational age at enrolment.

ANCA, antenatal care; DBS, dried blood spots; P, PrEP users; PrEP, pre-exposure prophylaxis; T, targeted arm participants only; U, universal arm participants only; $\mathrm{X}$, all study participants.

server. Data are transported via secure socket layer and only accessible by authenticated users. Only predesignated PrIMA staff have access to study data. Weekly reports, including performance indicators and summaries of severe adverse events, are generated to monitor study progress and troubleshoot problems. All data collected as part of this proposed research project will be made available without cost after registration to access or download files on a study-related website (URL to be determined) and agreement to the data sharing agreement after completion of primary study analyses.

\section{Patient and public involvement}

Two Community Advisory Boards (CABs) have been engaged to review the study aims, procedures and activities. The CABs include community leaders, educators, local ministry of health representatives, women and adolescents from the community. One CAB was established in Siaya County and one in Homa Bay County to ensure local acceptability of the study questions and procedures. The CABs meet at least two times per year to review study progress to date. Results of the study will be disseminated through peer-reviewed journals, presentations at relevant local and international conferences, to $\mathrm{MOH}$ representative including County and national HIV divisions, to the CABs engaged for support of the study and to participating partners and facility staff through facility-based meetings.

An EAP was convened to review recruitment, enrolment and potential social harms annually during the course of the study. The EAP reviews the study protocol, data collection schedule, recruitment, enrolment and retention data to ensure the study is achieving its objectives. The EAP is also responsible for reviewing any social harm events to ensure the safety of study participants. The EAP can recommend suspension or termination of the study due to serious concerns about subject's safety, inadequate performance, scientific or policy developments that impact the study or inadequate enrolment rates.

\section{Sample size}

Sample size was calculated based on a twofold difference in HIV incidence between study arms. Assuming a coefficient of variation $(\mathrm{k})$ of 0.2 , the study has $80 \%$ power to detect a twofold difference in annual HIV incidence (between $4 \%$ and $2 \%$ ) with 10 clinics per cluster and 200 women per 


\begin{tabular}{llllll}
\hline \multicolumn{5}{l}{ Table 3} & \multicolumn{5}{l}{ Sample size power calculations } \\
\hline & $\begin{array}{l}\text { HIV incidence } \\
\text { universal (\%) }\end{array}$ & $\begin{array}{l}\text { HIV incidence } \\
\text { targeted (\%) }\end{array}$ & $\begin{array}{l}\text { No. of } \\
\text { women } \\
\text { per clinic }\end{array}$ & $\begin{array}{l}\text { No. of } \\
\text { clinics } \\
\text { per arm }\end{array}$ & $\begin{array}{l}\text { Total no. } \\
\text { of women }\end{array}$ \\
\hline 0.2 & 4 & 2 & 50 & 27 & 2700 \\
\hline 0.2 & 4 & 2 & 100 & 15 & 3000 \\
0.2 & 4 & 2 & 150 & 12 & 3600 \\
0.2 & 4 & 2 & 200 & 10 & 4000 \\
0.2 & 4 & 2 & 250 & 9 & 4500 \\
0.2 & 4 & 2 & 300 & 8 & 4800 \\
0.2 & 4 & 1.3 & 200 & 6 & 2400 \\
\hline & & & & & \\
\hline
\end{tabular}

cluster (table 3). If targeted PrEP resulted in much better performance $(67 \%$ decrease rather than $50 \%)$, six clinics per cluster would be sufficient. Deriving our sample size from HIV incidence difference is conservative and enables ample statistical power to detect effects on other outcomes (such as proportion on PrEP and partner characteristics). Table 4 outlines implications of potential RCT outcomes illustrating the value of data regarding the two PrEP delivery models in scenarios with or without a significant difference in HIV incidence. Estimates suggest the targeted arm may have $20 \%$ of women receiving PrEP, while the universal PrEP arm may have 5\%-25\% of women requesting PrEP; however, these are speculative estimates.

If PrEP uptake is high, or high among women with greatest HIV risk, the study may not detect a difference in HIV incidence between PrEP delivery models. However, as outlined in the contingency table (table 4), for all scenarios, the study would yield important data on viable approaches for delivering PrEP in pregnancy and likely reveal a superior model in terms of the balance of effectiveness, safety, acceptability, feasibility and cost-effectiveness.

\section{Statistical methods and analysis}

The primary analysis will be intention to treat with HIV incidence compared between trial arms using generalised estimating equations (GEE) with a Poisson link (log person time since enrolment offset). The proportion of all women enrolled in the study who use PrEP (proportion of women exposed to PrEP and duration of PrEP exposure) will be compared between trial arms using GEE with a binomial link. The proportion with 'appropriate' use will be compared between arms using GEE with a binomial link. Appropriate PrEP use will be defined based on the risk assessment tool defined by Pintye et al including lifetime number of sexual partners, current partners HIV status and maternal syphilis test results in ANC. ${ }^{15}$ To assess the impact of differential loss to follow-up, we will conduct a sensitivity analysis to assess the robustness of results given missing outcomes and consider multiple imputation if necessary.

PrEP adherence will be measured among women on PrEP using DBS TFV drug levels. For each trial arm (10 clinic-cluster), a random subset of 220 DBS will be assessed. TFV drug levels will be compared by trial arm using GEE with a Gaussian link. PrEP duration will be computed for each woman based on self-report and pharmacy records and average duration compared between arms using GEE with a Gaussian link. Reported and confirmed partner with HIV status, proportions of partner HIV positive, unknown and HIV negative status and reported and confirmed partner ART use will be compared between arms using GEE with a binomial link.

Infant outcomes, including mean birth weight and serial weight-for-age z scores (WAZ), height-for-age z scores (HAZ) and weight-for-height $\mathrm{z}$ scores, will be compared between trial arms using GEE with a Gaussian link. The proportion preterm will be compared by PrEP exposure using GEE with a binomial link. PrEP-exposed infants will be compared with

Table 4 Contingency table demonstrating implications of potential RCT outcomes

Potential HIV

incidence outcome Other potential results

$\begin{array}{lll}\begin{array}{l}\text { Hypothesis } \\ \text { proved }\end{array} & \text { Targeted better. } & \begin{array}{l}\text { Fewer women on PrEP, } \\ \text { cost-effective and safe. }\end{array} \\ \begin{array}{l}\text { Hypothesis } \\ \text { opposed }\end{array} & \text { Universal better. } & \begin{array}{l}\text { Fewer women on PrEP, } \\ \text { cost-effective and safe. }\end{array}\end{array}$

Impact on programmes policy Programmatically relevant in high HIV prevalence regions data from study

Implement targeted PrEP.

Implement universal PrEP.

$\begin{array}{ll}\text { Universal too many } & \text { Refine universal strategy to } \\ \text { women on PrEP not cost } & \text { decrease cost and unnecessary }\end{array}$ effective.

Incidence low in both, Targeted more cost- Implement targeted PrEP. no difference. effective results in few on

PrEP.

Universal more cost- Implement universal PrEP. women on PrEP. 
Table 5 Stratified purposive sampling scheme and topics for qualitative interviews

\begin{tabular}{llllll}
\hline $\begin{array}{l}\text { Women: high } \\
\text { risk; no PrEP }\end{array}$ & $\begin{array}{l}\text { Women: low } \\
\text { risk; PrEP }\end{array}$ & $\begin{array}{l}\text { Women: high } \\
\text { adherence }\end{array}$ & $\begin{array}{l}\text { Women: high risk; } \\
\text { low adherence }\end{array}$ & $\begin{array}{l}\text { Male } \\
\text { partners }\end{array}$ & Community leaders \\
\hline Up to 20 & Up to 20 & Up to 20 & Up to 20 & Up to 15 & Up to 10 \\
\hline
\end{tabular}

Personal HIV risk perception Knowledge, attitudes and beliefs about PrEP

Partner communication strategies and behaviours

Barriers and facilitators of implementation

Knowledge, attitudes and beliefs about PrEP

Reasons for uptake/refusal of PrEP or HIV testing

Suggestions for improved delivery

Barriers and facilitators of PrEP use and adherence

PrEP, pre-exposure prophylaxis.

PrEP-unexposed infants for mean birth weight, WAZ and HAZ using GEE with a Gaussian link.

\section{Aim 2: qualitative methods}

Focus group discussions (FGDs) and in-depth interviews (IDI) will be used to evaluate barriers and facilitators of PrEP implementation, uptake and use among women, their male partners, healthcare providers and community and county leaders. Based on prior experience conducting similar qualitative research, data saturation will be reached with the sample sizes described in table $5 .^{25} 26$ All IDIs and FGDs will be performed in a private area. Consent will be obtained from participants to take notes and audio record the discussion. Interviews with women and male partners will focus on understanding how and why women make decisions to accept and adhere to PrEP and the feasibility of different PrEP use strategies. FGDs among healthcare workers will include those who are currently involved in offering PrEP or MCH services to understand logistical factors influencing healthcare worker ability and willingness to provide PrEP, specifically in the universal versus targeted strategies.

Discussions will be guided by a facilitator using a topic guide. Prior to the FGD, the facilitator will stress the importance of maintaining confidentiality within the group. Participants will receive unique identification numbers and will not be addressed by their real names so as to maintain confidentiality. Topics that generate the most discussion, participant attitudes, non-verbal gestures and interaction dynamics among group members will be documented by the moderator and note taker. Discussions will be conducted in English, Kiswahili or Luo depending on participant preferences. Thereafter, notes will be compared with audio-recordings to fill in missing information and transcribed to English (if necessary).

\section{Aim 3: health economics methods}

To evaluate the economic impact of providing PrEP in $\mathrm{MCH}$ settings, the incremental cost-effectiveness of the two approaches of PrEP delivery will be calculated per incident HIV infection and disability-adjusted life year averted. The base case costs of facility-based care will be estimated, and sensitivity analyses will be conducted. Cost data will be collected from a variety of sources including study records, publicly available data and in collaboration with the ministry of health. Cost data will include: (1) costs of all commodities used in the intervention; (2) labour costs for intervention workers; (3) average time clients spent with intervention including transportation/staff time/time for referrals; (4) local wages of target population; (5) rent; (6) maintenance; (7) volunteer activities; (8) user fees; (9) conversion rate of local currency (Kenyan shilling) to US dollars; (10) value of donated goods and services; and (11) other relevant costs, including training of providers and mobile van and fuel costs for community-based delivery. A discount rate of $3 \%$ will be used with sensitivity analysis of $0 \%-5 \%$.

Time and motion studies will be conducted to determine the time and resources necessary to provide PrEP counselling in pregnancy, monitoring and resupply. A single research assistant will collect time and motion data from at least three facilities in both the universal and targeted arm; time and motion observation may be done at additional facilities as needed until a typical time for PrEP counselling, initiation and dispensation can be estimated. Additionally, at each facility, a survey will be conducted, asking staff to estimate the amount of time it takes to conduct each step of the PrEP screening, counselling and dispensation processes and separating out any time for research procedures (such as informed consent). Discrepancies between the observed and self-reported time periods for activities will be discussed with staff. Observing multiple visits will allow estimation of the average time taken for each step; the time taken for research purposes (eg, data collection) will be noted separately from the estimated time needed for clinical services. Through time and motion studies, the number of participants who could be supported by a clinic will be estimated.

For analysis taking, the societal perspective, costs to clients in terms of lost time, wages, childcare and other relevant opportunity costs will be collected. Participants will be asked what expenses and opportunity costs they incurred to receive the interventions in the surveys already planned for the project. Information on the average cost of medical care in Kenya associated with pregnancy, postpartum, HIV infection and AIDS will be collected through literature review.

Cost data will be used with study results and mathematical models of HIV transmission to estimate the 
population-level effectiveness and cost-effectiveness of the intervention. The models will use deidentified study data.

\section{Ethics}

The PrIMA study was registered at clinicaltrials.gov. Changes to the protocol will be reviewed by both the University of Washington Institutional Review Board (IRB) and Kenyatta National Hospital Ethics and Research Committe (ERC) prior to implementation. Any changes to the protocol will be communicated to coinvestigators and study staff through change memos, and the protocol at clinicaltrials.gov will be updated.

Acknowledgements Felix Abuna Otieno, Peter Onyango and Ben Ochieng for their work on integrating the PrIMA study into all study facilities and ensuring the standard operating procedures are implemented. Daniel Odinga for the development laboratory procedures and ensuring quality control procedures are followed. Marwa Motongori and Salphine Watoyi for supporting the implementation of data collection measures and ensuring data quality daily. Emma Mukenyi and Rita Akumu for ensuring all study staff and facilities have the supplies required to implement study procedures. The PrIMA Community Advisory Boards in Siaya and Homa Bay County for their thoughtful feedback and support on all aspects of protocol development and implementation.

Contributors GJ-S, JMB, JK and BAR designed the cluster randomised trial. JCD, JK, NM, BAR, RB, ADW, GO, JMB and GJ-S developed the study protocol. GJ-S and JMB are multiple principal investigators. JK is the country principal investigator, JCD is the project director, BAR oversaw development of the statistical analysis plan, $\mathrm{GO}$ developed the qualitative methods and RB developed the cost effectiveness analysis plan. JK, JP, BAR, RB, ADW, GO, JMB and GJ-S provided scientific expertise. JCD, JP, NM and LG oversee data collection and data management activities. JCD, JP, NM and LG oversee implementation of the study procedures. JP oversees all laboratory procedures. All authors have contributed to the development of this manuscript, have read and approved the final version for publication.

Funding This work was funded by the National Institutes of Allergy and Infectious Disease (NIAID), grant number R01 Al125498.

Competing interests None declared.

Patient consent for publication Not required.

Ethics approval The study protocol was approved by the IRB at the University of Washington (STUDY00000438) and the ERC at Kenyatta National Hospital (P73/02/2017).

Provenance and peer review Not commissioned; externally peer reviewed.

Open access This is an open access article distributed in accordance with the Creative Commons Attribution Non Commercial (CC BY-NC 4.0) license, which permits others to distribute, remix, adapt, build upon this work non-commercially, and license their derivative works on different terms, provided the original work is properly cited, appropriate credit is given, any changes made indicated, and the use is non-commercial. See: http://creativecommons.org/licenses/by-nc/4.0/.

\section{REFERENCES}

1. Drake AL, Wagner A, Richardson B, et al. Incident HIV during pregnancy and postpartum and risk of mother-to-child HIV transmission: a systematic review and meta-analysis. PLoS Med 2014;11:e1001608.

2. Kinuthia J, Drake AL, Matemo D, et al. HIV acquisition during pregnancy and postpartum is associated with genital infections and partnership characteristics. AIDS 2015;29:2025-33.

3. Thomson KA, Hughes J, Baeten JM, et al. Increased risk of HIV acquisition among women throughout pregnancy and during the postpartum period: a prospective per-coital-act analysis among women with HIV-infected partners. J Infect Dis 2018;218:16-25.
4. Dunn DT, Newell ML, Ades AE, et al. Risk of human immunodeficiency virus type 1 transmission through breastfeeding. The Lancet 1992;340:585-8.

5. Lockman S, Creek T. Acute maternal HIV infection during pregnancy and breast-feeding: substantial risk to infants. J Infect Dis 2009;200:667-9.

6. Fonner VA, Dalglish SL, Kennedy CE, et al. Effectiveness and safety of oral HIV preexposure prophylaxis for all populations. AIDS 2016;30:1973-83.

7. Mofenson LM, Baggaley RC, Mameletzis I. Tenofovir Disoproxil Fumarate Safety for Women and their Infants during Pregnancy and Breastfeeding: Systematic Review. AIDS 2016;31(2):213-32.

8 Wang L, Kourtis AP, Ellington S, et al. Safety of tenofovir during pregnancy for the mother and fetus: a systematic review. Clin Infect Dis 2013;57:1773-81.

9 Mugwanya KK, Hendrix CW, Mugo NR, et al. Pre-exposure prophylaxis use by breastfeeding HIV-uninfected women: a prospective short-term study of antiretroviral excretion in breast milk and infant absorption. PLoS Med 2016;13:e1002132.

10 Ministry of Health NASCOP. Guidelines on Use of Antiretroviral Drugs for Treating and Preventing HIV Infection in Kenya 2016. Nairobi, Kenya: NASCOP, 2016.

11. World Health Organization. WHO Technical brief: Preventing HIV during pregnancy and breastfeeding in the context of pre-exposure prophylaxis (PrEP). Geneva: World Health Organization, 2017.

12. Kuhns LM, Hotton AL, Schneider J, et al. Use of Pre-exposure Prophylaxis (PrEP) in young men who have sex with men is associated with race, sexual risk behavior and peer network size. AIDS Behav 2017;21:1376-82.

13. Rendina HJ, Whitfield TH, Grov C, et al. Distinguishing hypothetical willingness from behavioral intentions to initiate HIV pre-exposure prophylaxis (PrEP): Findings from a large cohort of gay and bisexual men in the U.S. Soc Sci Med 2017;172:115-23.

14. Ridgway JP, Almirol EA, Bender A, et al. Which patients in the emergency department should receive preexposure prophylaxis? Implementation of a predictive analytics approach. AIDS Patient Care STDS 2018;32:202-7.

15. Pintye J, Drake AL, Kinuthia J, et al. A risk assessment tool for identifying pregnant and postpartum women who may benefit from preexposure prophylaxis. Clin Infect Dis 2017;64:751-8.

16. Ministry of Health NACC. Kenya AIDS Response Progress Report 2016. 2016.

17. Kenya National Bureau of Statistics, Ministry of Health, National AIDS Control Council, Kenya Medical Research Institute, National Council for Population and Development, The DHS Program, ICF International. Kenya Demographic and Health Survey 2014. Nairobi, Kenya, 2015.

18. Ivers NM, Halperin IJ, Barnsley J, et al. Allocation techniques for balance at baseline in cluster randomized trials: a methodological review. Trials 2012;13:120.

19. Thirumurthy $\mathrm{H}$, Masters $\mathrm{SH}$, Mavedzenge SN, et al. Promoting male partner HIV testing and safer sexual decision making through secondary distribution of self-tests by HIV-negative female sex workers and women receiving antenatal and post-partum care in Kenya: a cohort study. Lancet HIV 2016;3:e266-74.

20. Kosack CS, de Kieviet W, Bayrak K, et al. Evaluation of the Nova StatSensor $₫$ Xpress(TM) Creatinine point-of-care handheld analyzer. PLoS One 2015;10:e0122433.

21. WHO Multicentre Growth Reference Study Group. WHO Child Growth Standards based on length/height, weight and age. Acta Paediatr Suppl 2006;450:76-85.

22. Anderson PL, Liu AY, Castillo-Mancilla JR, et al. Intracellular tenofovir-diphosphate and emtricitabine-triphosphate in dried blood spots following directly observed therapy. Antimicrob Agents Chemother 2018;62.

23. Castillo-Mancilla JR, Zheng JH, Rower JE, et al. Tenofovir, emtricitabine, and tenofovir diphosphate in dried blood spots for determining recent and cumulative drug exposure. AIDS Res Hum Retroviruses 2013;29:384-90.

24. Harris PA, Taylor R, Thielke R, et al. Research electronic data capture (REDCap)-a metadata-driven methodology and workflow process for providing translational research informatics support. J Biomed Inform 2009;42:377-81.

25. Morse JM. Determining Sample Size. Qual Health Res 2000;10:3-5.

26. Marshall MN. Sampling for qualitative research. Fam Pract 1996;13:522-6. 